\title{
A search for massive ultra-compact dwarf galaxies in the Centaurus galaxy cluster ${ }^{\star}$ (Research Note)
}

\author{
S. Mieske ${ }^{1}$, M. Hilker ${ }^{2}$, I. Misgeld ${ }^{2,3}$, A. Jordán ${ }^{4,5}$, L. Infante ${ }^{4}$, and M. Kissler-Patig ${ }^{2}$ \\ 1 European Southern Observatory, Alonso de Cordova 3107, Vitacura, Santiago, Chile \\ e-mail: smieske@eso.org \\ 2 European Southern Observatory, Karl-Schwarzschild-Strasse 2, 85748 Garching bei München, Germany \\ 3 Argelander Institut für Astronomie, Auf dem Hügel 71, 53121 Bonn, Germany \\ 4 Departamento de Astronomía y Astrofísica, Pontificia Universidad Católica de Chile, Casilla 306, Santiago 22, Chile \\ 5 Harvard-Smithsonian Center for Astrophysics, 60 Garden St., Cambridge, MA 02138, USA
}

Received 29 October 2008 / Accepted 27 January 2009

\section{ABSTRACT}

\begin{abstract}
Context. In a previous paper, we initiated a search for ultra-compact dwarf galaxies (UCDs) in the Centaurus galaxy cluster, resulting in the discovery of 27 compact objects with $-12.2<M_{V}<-10.9$ mag. Our overall survey completeness was $15-20 \%$ within $120 \mathrm{kpc}$ projected clustercentric distance.

Aims. To constrain the luminosity distribution of the brightest UCDs in Centaurus in a more accurate way, we continue our search by improving substantially our survey completeness specifically for brightnesses of $M_{V}<-12 \mathrm{mag}\left(V_{0}<21.3 \mathrm{mag}\right)$.

Methods. Using VIMOS at the VLT, we obtained low resolution spectra of 400 compact objects with $19.3<V_{0}<21.3$ mag $\left(-14<M_{V}<-12 \mathrm{mag}\right.$ at the Centaurus distance) in the central $25^{\prime}$ of the Centaurus cluster, which corresponds to a projected radius of $\sim 150 \mathrm{kpc}$. Our survey had complete area coverage within a radius of $\sim 120 \mathrm{kpc}$.

Results. For $94 \%$ of the sources included in the masks, we measure successfully a redshift. Because of incompleteness in the slit assignment, our final completeness in the area surveyed is 52\%. Among our targets, we find three new UCDs in the magnitude range $-12.2<M_{V}<-12 \mathrm{mag}$, hence at the faint limit of our survey. For one of these targets HST WFPC2 imaging is available, yielding a size estimate of $r_{\mathrm{h}} \lesssim 8-9 \mathrm{pc}$. At $95 \%$ confidence, we can reject the hypothesis that in the area surveyed there are more than 2 massive UCDs with $M_{V}<-12.2 \mathrm{mag}$ and $r_{\text {eff }} \lessgtr 70 \mathrm{pc}$. Hence our survey confirms the extreme rarity of massive UCDs. We find that the radial distributions of Centaurus and Fornax UCDs with respect to their host clusters' centres agree within the $2 \sigma$ level.
\end{abstract}

Key words. galaxies: clusters: individual: Centaurus - galaxies: dwarf - galaxies: fundamental parameters - galaxies: nuclei galaxies: star clusters

\section{Introduction}

A new class of compact stellar systems called "ultra-compact dwarf galaxies" (UCDs, Phillipps et al. 2001) has been established (Hilker et al. 1999; Drinkwater et al. 2000, 2003; Hasegan et al. 2005; Jones et al. 2006; Mieske et al. 2007; Firth et al. 2007; Misgeld et al. 2008). UCDs are characterised by typical luminosities of $-13.5<M_{V}<-11.0 \mathrm{mag}$, half-light radii of $10<r_{\mathrm{h}}<100 \mathrm{pc}$, and masses of $2 \times 10^{6}<m<10^{8} M_{\odot}$. An intriguing finding is that, on average, the dynamical $M / L$ ratios of UCDs are about twice as high as those of Galactic globular clusters of comparable metallicity (e.g., Hasegan et al. 2005; Hilker et al. 2007; Evstigneeva et al. 2007; Rejkuba et al. 2007; Mieske et al. 2008a). Indications exist that $M / L$ ratios may be somewhat higher for UCDs in Virgo than in Fornax (e.g., Hasegan et al. 2005; Hilker et al. 2007; Evstigneeva et al. 2007), which could be explained by differences in age, stellar mass function, or dark matter content.

To broaden the environmental baseline of UCD research, we have embarked on UCD searches in the Centaurus galaxy cluster (Mieske et al. 2007) and the Hydra I galaxy cluster (Misgeld et al. 2008), based on data obtained with VIMOS at the VLT

\footnotetext{
^ Based on observations obtained in service mode at the VLT (programme 080.B-0307).
}

(program 076.B-0293). From the UCD search in Centaurus, we have found 27 compact objects with radial velocities consistent with them being members of Centaurus, covering an absolute magnitude range $-12.2<M_{V}<-10.9 \mathrm{mag}$. Their distribution in magnitude and space was found to be consistent with that of the GC population. We found no very luminous UCDs with $-13.5<M_{V}<-12.2 \mathrm{mag}$ as found in the Virgo and Fornax cluster (Jones et al. 2006; Chilingarian \& Mamon 2008; Drinkwater et al. 2000), which may be due to the moderate overall completeness of 15-20\% within $120 \mathrm{kpc}$ : the survey covered about $50-60 \%$ of the total area within $120 \mathrm{kpc}$, within which only $30 \%$ of the photometrically selected sources were observed, because of too high candidate density (see Mieske et al. 2007; and Fig. 2).

In this Research Note, we report on the second part of our search for UCDs in Centaurus with VIMOS (program 380.B0207), in which we have attempted to constrain more accurately their luminosity distribution at the bright end. We increase the area coverage and focus on the bright luminosity regime $M_{V}<-12$ mag. Our medium-term strategy is to derive dynamical mass estimates for UCDs beyond the Fornax and Virgo clusters to investigate in depth whether average $M / L$ ratios of UCDs systematically vary with environment. Technically, groundbased medium-to-high resolution spectroscopy $(R \sim 10000)$ is 
possible only for objects with $V \lesssim 21.5 \mathrm{mag}$ (e.g., Mieske et al. 2008a; Chilingarian et al. 2008), which translates to a feasibility limit of $M_{V, 0} \lesssim-12 \mathrm{mag}$ at the distance of the Centaurus cluster $((m-M) \simeq 33.3 \mathrm{mag}$, Mieske et al. 2005). This is another driver for focusing on the bright luminosity regime $M_{V}<-12 \mathrm{mag}$.

\section{The data}

The data for this publication were obtained in service mode with the VIsible MultiObject Spectrograph VIMOS (Le Fevre et al. 2003) mounted on UT3 Melipal at the VLT (programme 380.B0207). VIMOS allows simultaneous observing of 4 quadrants, each of dimension $7^{\prime} \times 8^{\prime}$ and separated by about $2^{\prime}$. We observed four multi-object spectroscopy (MOS) pointings close to NGC 4696, the central galaxy of the main cluster component Cen30 (see Fig. 2). Two of those pointings had already been targeted as part of our previous observing program in Period 76 (Mieske et al. 2007). However, given the slit allocation completeness of about $30 \%$ in that run, we reobserved these pointings. We observed two further pointings that were slightly offset from the others to ensure that the effect of chip gaps was minimized, increasing the area coverage within $\sim 120 \mathrm{kpc}$ to almost $100 \%$ (see Figs. 2 and 3). Within the four pointings, a slit could be allocated for $54 \%$ of the photometrically selected objects (see Sect. 2.2).

\subsection{Candidate selection}

The candidates for our search for bright UCDs were selected from the VIMOS pre-imaging in the $V$ and $R$ filters which were taken under clear conditions. Prior to applying any selection, we matched the detections in V,R to the catalog of well calibrated FORS photometry (Mieske et al. 2005) of the central Centaurus cluster in V and I, whose areas overlap with the VIMOS preimaging. From this matching we were able to verify that the $V$-band VIMOS zeropoints available from the ESO QC web pages $^{1}$ for the date of the pre-imaging (17-01-2008) were accurate to within $0.03-0.05$ mag.

For dereddening the apparent magnitudes we used data from Schlegel et al. (1998). To select sources as compact object candidates, we defined three criteria regarding size, colour, and luminosity:

1. unresolved in the VIMOS pre-imaging date (as judged by the SExtractor star-galaxy separator, Bertin \& Arnouts 1996). At the distance of the Centaurus cluster (45 Mpc, Mieske et al. 2005), the typical PSF $F W H M$ of $0.85^{\prime \prime}$ corresponds to $\approx 190$ pc. In Fig. 1, we present SExtractor star-galaxy separator values measured for simulated seeing convolved UCD images that had been superimposed on our VIMOS pre-images. The simulations were based on the structural parameters of UCD3 (Hilker et al. 2007), assuming a Centaurus cluster distance of $45 \mathrm{Mpc}$ (Mieske et al. 2005), and a seeing of $0.85^{\prime \prime}(\approx 190 \mathrm{pc})$. UCD3 itself has $r_{\text {eff }} \sim 90$ pc. To simulate UCDs of a range of sizes, we scaled the surface brightness profile of UCD3 to smaller/larger radii. The plot shows that the SExtractor star classifier value flips from "star" to "galaxy" for $r_{\mathrm{eff}} \gtrsim 70 \mathrm{pc}$. Our size selection criterion hence encompasses all known UCDs, except the two most massive ones, each of which have $r_{\mathrm{eff}} \simeq 100 \mathrm{pc}$ (Evstigneeva et al. 2008). This corresponds to $\sim 95 \%$ of

\footnotetext{
1 http://www . eso.org/observing/dfo/quality/index_ vimos.html
}

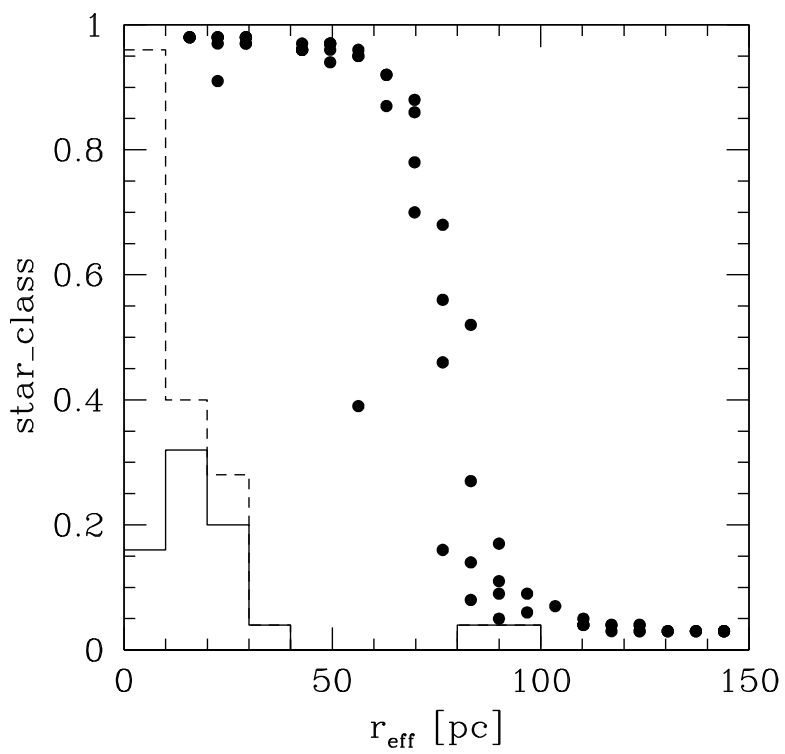

Fig. 1. This plot illustrates the upper limit to UCD effective radius that our candidate selection criterion for unresolved sources corresponds to. The $y$-axis shows the SExtractor star-classifier value (Bertin \& Arnouts 1996), which is defined to be 1 for an unresolved source ("star"), and 0 for a resolved source ("galaxy"). The SExtractor star-classifier value flips from "star" to "galaxy" for $r_{\text {eff }} \gtrsim 70$ pc. The solid histogram is the size distribution of known UCDs with $M_{V}<-12$ mag, arbitrarily normalised. The dashed histogram is the size distribution of all UCDs (hence $M_{V}<-11 \mathrm{mag}$ ), normalised by the same factor as the dashed histogram.

all known UCDs, and $\simeq 85 \%$ of known UCDs with $M_{V}<$ $-12 \mathrm{mag}^{2}$;

2. with de-reddened colours of $0.42<(V-R)_{0}<0.9$ mag. This $(V-R)_{0}$ range corresponds to a $(V-I)_{0}$ range from 0.65 to 1.50 mag (see Fig. 3), which is the colour range typically covered by GCs (e.g. Gebhardt \& Kissler-Patig 1999; Larsen et al. 2001; Kundu \& Whitmore 2001). This also includes the colours of UCDs discovered in the first part of our search (Mieske et al. 2007). Figure 3 shows the directly measured $(V-R)_{0}$ colours, and the corresponding $(V-I)_{0}$ scale. This scale is derived from matching the $V$ and $R$ preimaging photometry of unresolved sources with $V, I$ photometry from spatially overlapping FORS data (see above; Mieske et al. 2005). The derived scaling in apparent magnitude space is

$(V-I)=-0.163+1.911 \times(V-R)$

with an rms of $0.11 \mathrm{mag}$;

3. with dereddened apparent magnitudes $19.3<V_{0}<21.3 \mathrm{mag}$ $\left(-14<M_{V}<-12 \mathrm{mag}\right)$. The faint magnitude cut is more than a magnitude brighter than in the first part of our search (Mieske et al. 2007).

\subsection{Spectroscopic observations}

Within our 16 masks ( 4 pointings $\times 4$ quadrants), the VIMOS mask creation software VMMPS enabled the allocation of slits for 412 objects (minimum slit length $6^{\prime \prime}$ ), compared to a total of 766 photometrically selected sources. We were able to measure redshifts for 389 of those 412 sources. Our completeness

\footnotetext{
2 A complementary observing campaign targeting canonical dwarf galaxies - including resolved UCD candidates - in Centaurus has been approved for ESO observing period P83.
} 


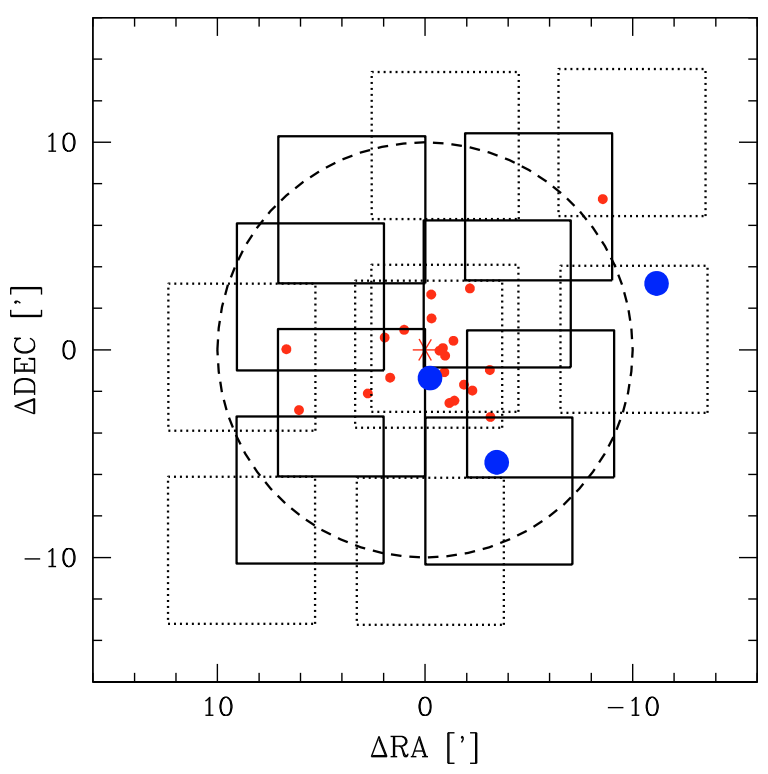

Fig. 2. Map of the central Centaurus cluster. The relative coordinates are with respect to NGC 4696, the central galaxy of the Cen30 subcluster. The dotted squares indicate the VIMOS pointing observed in P76 (Mieske et al. 2007). Note that the VIMOS field-of-view consists of four quadrants. The small (red) dots indicate the UCDs detected in that survey, covering a magnitude range $-12.2<M_{V}<-10.8 \mathrm{mag}$. For the new run in P80 which is reported upon in this paper, we re-observed the dotted pointings (see text) with two masks per quadrant, added two more pointings (solid squares), and focused only on $M_{V}<-12$ mag. The large (blue) dots indicate the three UCDs found in this new run (see Sect. 3). The dashed circle indicates a projected clustercentric distance of $\sim 120 \mathrm{kpc}$, within which most Fornax and Virgo UCDs are found (see Thomas et al. 2008, for a sample of fainter intra-cluster UCDs in Fornax at larger projected radii).

in the entire area surveyed was hence $51 \%$ (Fig. 4), about three times higher than in the previous part of our survey (Mieske et al. 2007). Within the central $120 \mathrm{kpc}$, the completeness was $52 \%$, only marginally higher.

We used the medium resolution MR grism with the order-sorting filter GG475. This covers the wavelength range from 4800 to $10000 \AA$ at a dispersion of $2.5 \AA$ per pixel. The average seeing for the spectroscopic observations was around $0.8^{\prime \prime}$, at a slit width of $1.0^{\prime \prime}$. With a pixel scale of $0.2^{\prime \prime}$, the instrumental resolution $(F W H M)$ is $10-12 \AA$, corresponding to a velocity resolution of $\sim 600 \mathrm{~km} \mathrm{~s}^{-1}$. For each pointing, the total exposure time was $2100 \mathrm{~s}$. Arc-lamp exposures for wavelength calibration were attached to each science exposure.

\subsection{Data reduction}

For the data reduction from $2 \mathrm{D}$ raw spectra to wavelength calibrated 1D spectra, we used the recipe vmmosobsstare provided by the ESO VIMOS pipeline ${ }^{3}$. This recipe performs bias subtraction, flat field division, wavelength calibration, and spectrum extraction. Figure 5 shows four examples of calibrated 1D spectra.

The radial velocity measurements of the calibrated 1D spectra were performed by cross-correlation using the IRAF task fXcor (Tonry \& Davis 1979) in the RV package. As a template in the cross-correlation, we used a synthetic spectrum created to

\footnotetext{
3 http://www.eso.org/projects/dfs/dfs-shared/web/ vimos/vimos-pipe-recipes.html
}

resemble a typical early-type galaxy (Quintana et al. 1996). This template has proven to be the most reliable for this type of radial velocity surveys (e.g., Mieske et al. 2004; Misgeld et al. 2008). For a measurement to be accepted as reliable, we ensured that the cross-correlation confidence value $R$ was higher than 5.5. We then repeated our execution of fxcor for those spectra for which $R<5.5$ was achieved in the first execution, and accepted $v_{\text {rad }}$ measurements for those that showed clearly identifiable crosscorrelation peaks (see Fig. 5 for an example). For more than $90 \%$ of our observed sources, we could reliably measure a redshift (see Fig. 3). The radial velocity measurement errors were of the order $50-100 \mathrm{~km} \mathrm{~s}^{-1}$. As a cluster membership criterion, we required that $1750<v_{\text {rad }}<5550 \mathrm{~km} \mathrm{~s}^{-1}$, excluding both foreground stars and background galaxies.

\section{Results}

Figure 6 shows a radial velocity histogram of the 389 sources with measured redshifts. Of these 389 sources, 380 are foreground stars, and six objects are background galaxies with $9400<v_{\text {rad }}<41000 \mathrm{~km} \mathrm{~s}^{-1} 4$. Only three objects are members of the Centaurus cluster. At the cluster's distance modulus $((m-M)=33.3 \mathrm{mag}$, Mieske et al. 2005), they cover the magnitude range $-12.2<M_{V}<-12.0$ (Fig. 3), at the faint limit of our survey. Table 1 shows the properties of these three confirmed UCDs. We list their $V_{0}$ and $(V-R)_{0}$ magnitudes, their radial velocities and errors, and the confidence level $R$ of the radial velocity measurement. The estimated $(V-I)_{0}$ colour is also given, as derived from Eq. (1). For one of the UCDs, CCOS J192.20041.334, archival HST imaging in the $F 555 W$ filter (WFPC2, Proposal 5956, PI Sparks) was available. To measure its size, we used the program KINGPHOT (Jordán et al. 2004, 2005), which we had already successfully applied to measure the half-light radii $r_{\mathrm{h}}$ of GCs in Virgo and Fornax, and UCDs in Centaurus (Jordán et al. 2005, 2007; Mieske et al. 2007). From this fit, we derived a $2 \sigma$ upper limit to the projected half-light radius of 0.43 WFPC2 wide-field pixel, corresponding to $r_{\mathrm{h}} \lesssim 8-9 \mathrm{pc}$ at the assumed distance modulus of $33.3 \mathrm{mag}$.

We note that we discovered no UCD in the bright luminosity regime $-13.5 \lesssim M_{V} \lesssim-12.2 \mathrm{mag}$, within which also only a few UCDs have been found in Fornax and Virgo. This confirms the rarity of these extreme objects. Given our overall survey completeness of $\sim 50 \%$ and assuming a Poisson distribution for UCD number counts, we can exclude at a $95 \%$ confidence level the existence of more than two UCDs with $M_{V}<-12.2$ mag and $r_{\mathrm{eff}}<70 \mathrm{pc}$, within $120 \mathrm{kpc}$ of NGC 4696.

In spite of the lack of such very bright UCDs, there is now a total of eight confirmed UCDs with $M_{V}<-12$ mag in Centaurus (this paper, Mieske et al. 2007), comparable to the numbers in Virgo/Fornax (Jones et al. 2006; Firth et al. 2007). Seven of those eight sources belong to the main cluster Cen30. Given our completeness of $\sim 50 \%$ within the central $120 \mathrm{kpc}$ of Cen30, we can constrain the true number of UCDs with $M_{V}<-12$ mag in that area to be $14 \pm 5$. How does this compare to the number of GCs expected by extrapolating a Gaussian globular cluster luminosity function to $M_{V}<-12 \mathrm{mag}$ ? For the central Cen30 galaxy NGC 4696, we would expect a total of $\sim 14000 \mathrm{GCs}$, adopting a specific frequency of 7.3 (Mieske et al. 2005) and an absolute magnitude of $M_{V}=-23.2 \mathrm{mag}$ (Misgeld et al. 2009) for NGC 4696. Since we observed within a radius of $120 \mathrm{kpc}$, we assume that we include most of the GC systems (e.g.,

\footnotetext{
4 The list of foreground stars and background galaxies including their coordinates is available upon request from smieske@eso.org
} 

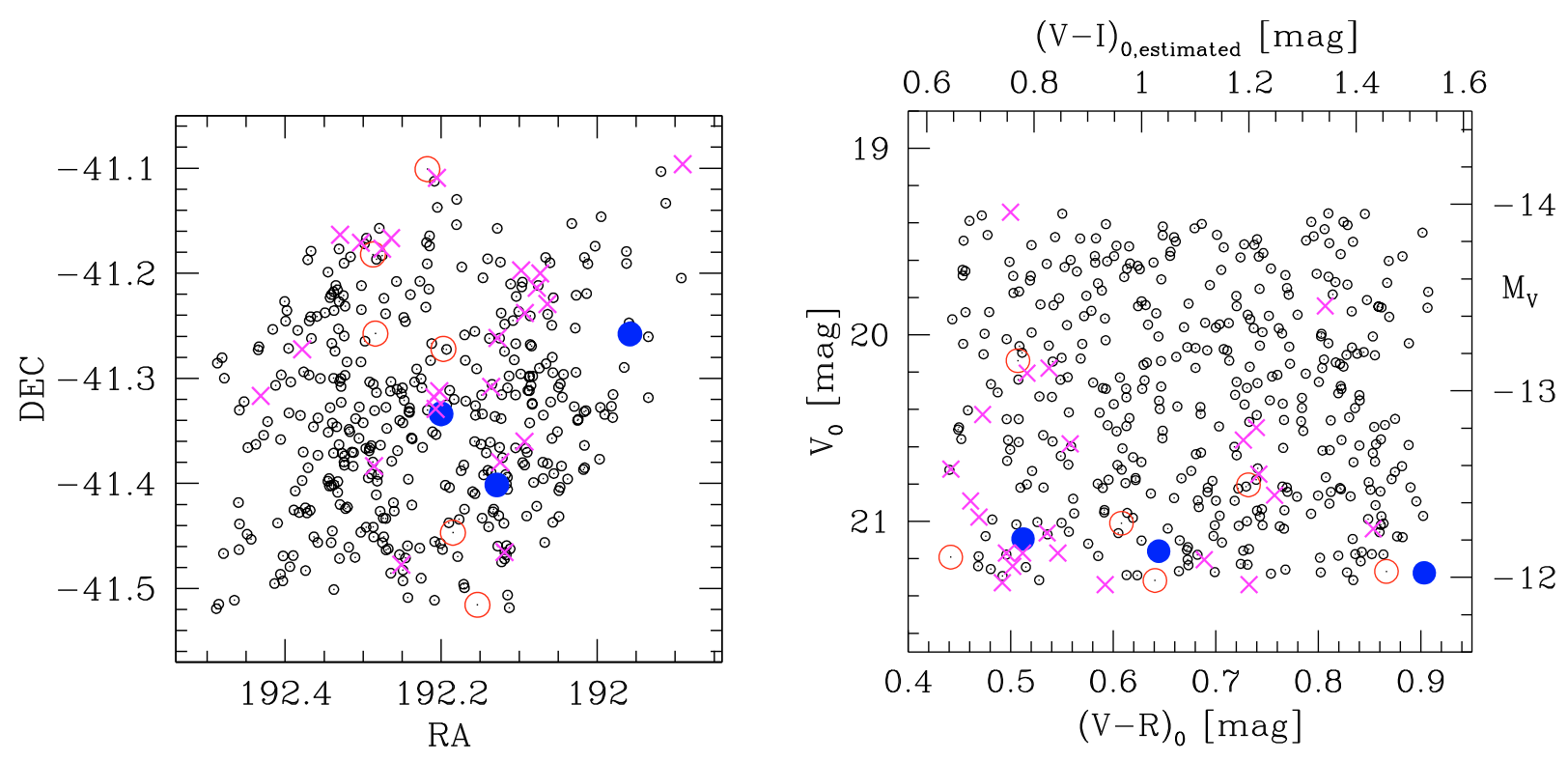

Fig. 3. Map (left) and CMD (right) of the objects observed in the VIMOS P80 run. Dots indicate all objects to which a slit was assigned. Small (black) circles indicate foreground stars. Large (red) open circles indicate background sources $\left(v_{\text {rad }}>6000 \mathrm{~km} \mathrm{~s}^{-1}\right)$. Magenta crosses mark objects for which no radial velocity could be measured. Filled (blue) circles indicate objects with radial velocities in the Centaurus cluster range, hence the UCDs found. In the CMD, the upper $x$-axis shows the approximate scale of $(V-I)_{0}$ colours. The scaling of $(V-R)$ with $(V-I)$ was derived from matching the $V, R$ pre-imaging photometry with spatially overlapping FORS $V$, I photometry (Mieske et al. 2005).
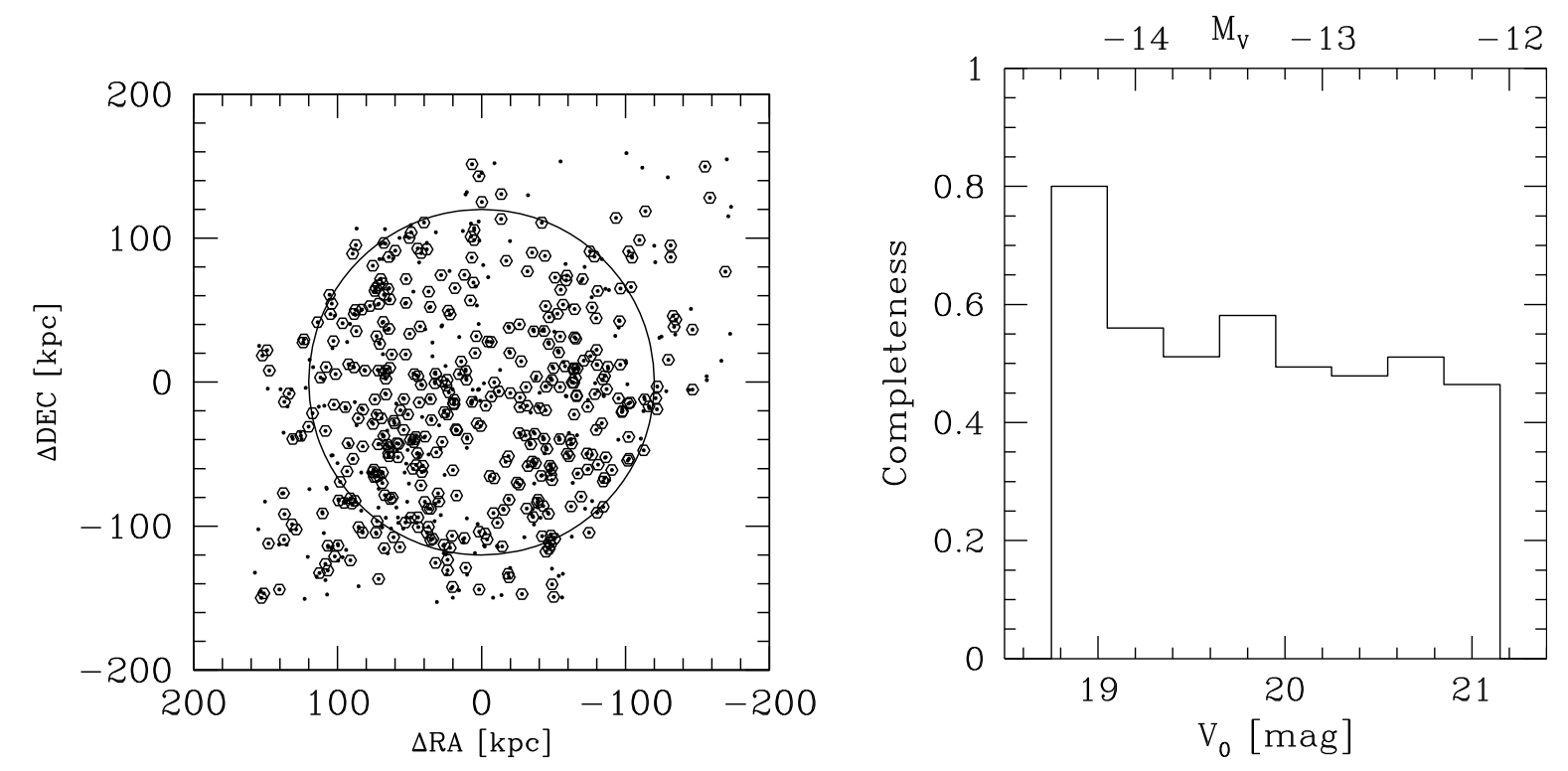

Fig. 4. Completeness of our survey. Left panel: plotted are all photometrically selected objects (small dots) and successfully observed objects (small hexagons). Note the difference to Fig. 3, where only objects included in the masks are plotted. The circle indicates a projected radius of $\sim 120 \mathrm{kpc}$ at the Centaurus cluster distance. Right panel: ratio of successfully observed objects to all photometrically selected objects within the central $120 \mathrm{kpc}$, as a function of magnitude.

Table 1. Properties of the 3 massive UCDs detected in our survey, ordered by magnitude. Errors are given in parentheses. "CCOS" in the object identifier stands for Centaurus Compact Object Survey, see also Mieske et al. (2007). The last column gives a $2 \sigma$ upper limit to the half-light radius in pc estimated from HST WFPC2 archival imaging.

\begin{tabular}{c|cccccccc}
\hline \hline ID & RA $(\mathrm{J} 2000)$ & Dec $(\mathrm{J} 2000)$ & $V_{0}$ & $(V-R)_{0}$ & $(V-I)_{0, \text { estimated }}$ & $v_{\text {rad }}\left[\mathrm{km} \mathrm{s}^{-1}\right]$ & $R$ & $r_{\mathrm{h}}[\mathrm{pc}]$ \\
\hline CCOS J192.129-41.401 & $12: 48: 30.88$ & $-41: 24: 04.96$ & 21.15 & 0.51 & 0.77 & $2650(47)$ & 8.06 & \\
CCOS J192.200-41.334 & $12: 48: 48.01$ & $-41: 20: 01.52$ & 21.22 & 0.64 & 1.03 & $2061(76)$ & 5.79 & $\lesssim 8-9$ \\
CCOS J191.958-41.258 & $12: 47: 49.93$ & $-41: 15: 28.04$ & 21.34 & 0.90 & 1.52 & $3185(60)$ & 5.94 & \\
\hline
\end{tabular}

Rhode \& Zepf 2001, showed that the GC system of NGC 4472, the most luminous early-type galaxy in Virgo, extends to $\sim 80 \mathrm{kpc})$. Adopting an absolute turnover magnitude of
$M_{V}=-7.4$ mag (Kundu \& Whitmore 2001) and a GCLF width of 1.35 mag (Jordán et al. 2006, 2007), the expected number of GCs with $M_{V}<-12$ mag is about 5. Although being on the low 

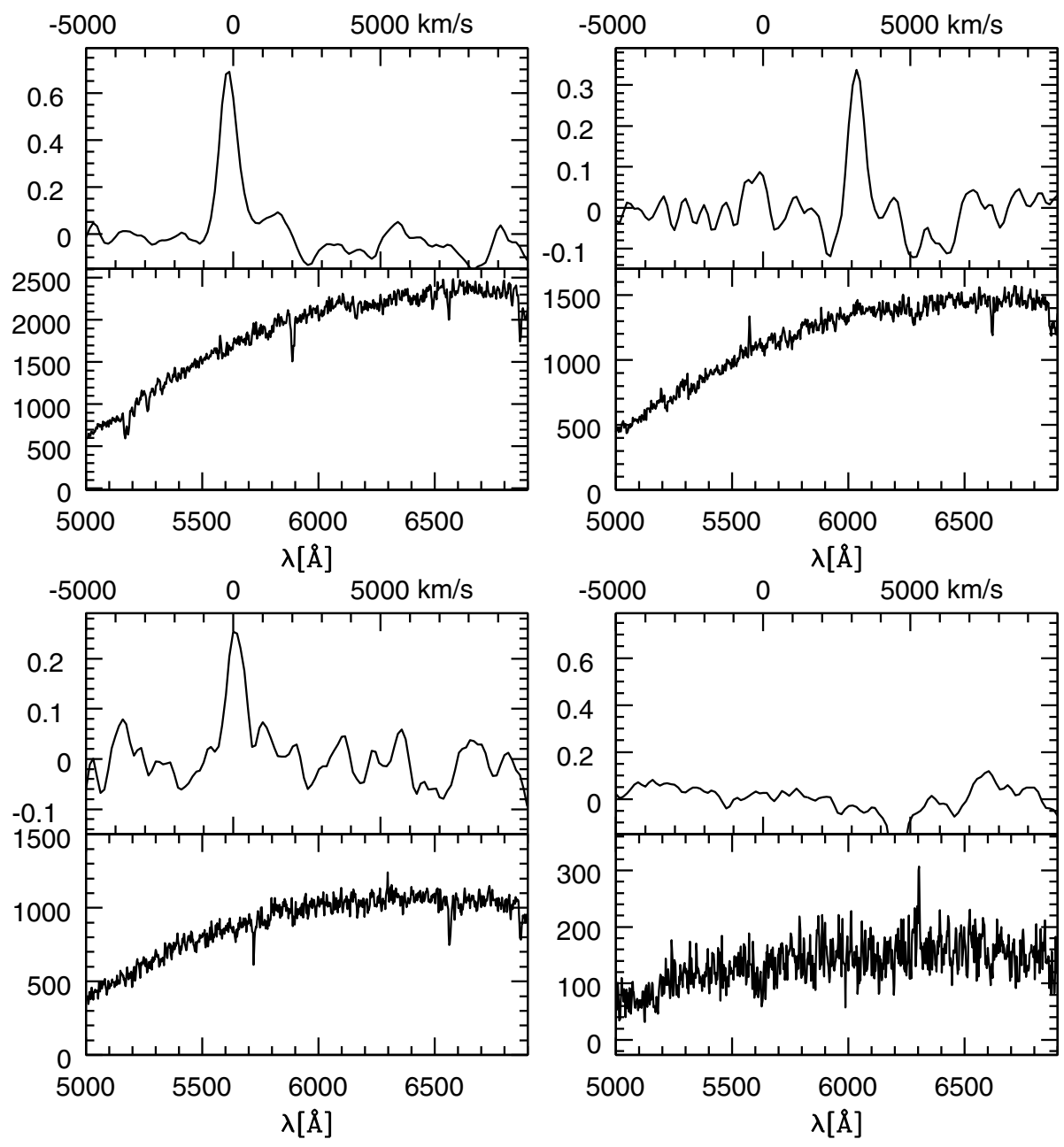

Fig. 5. Four example spectra of our VIMOS dataset, with the corresponding crosscorrelation results for radial velocity measurement indicated on top. $Y$-axis units are flux in ADU for the bottom plots, and crosscorrelation height $h$ for the top plots. Objects from upper left to bottom right: a foreground star with cross-correlation confidence level $R>5.5$; one of the three Centaurus cluster members (CCOS J192.129-41.401), also with $R>5.5$; a foreground star with $R=5.0$, whose radial velocity measurement was accepted; a source for which no radial velocity could be measured.
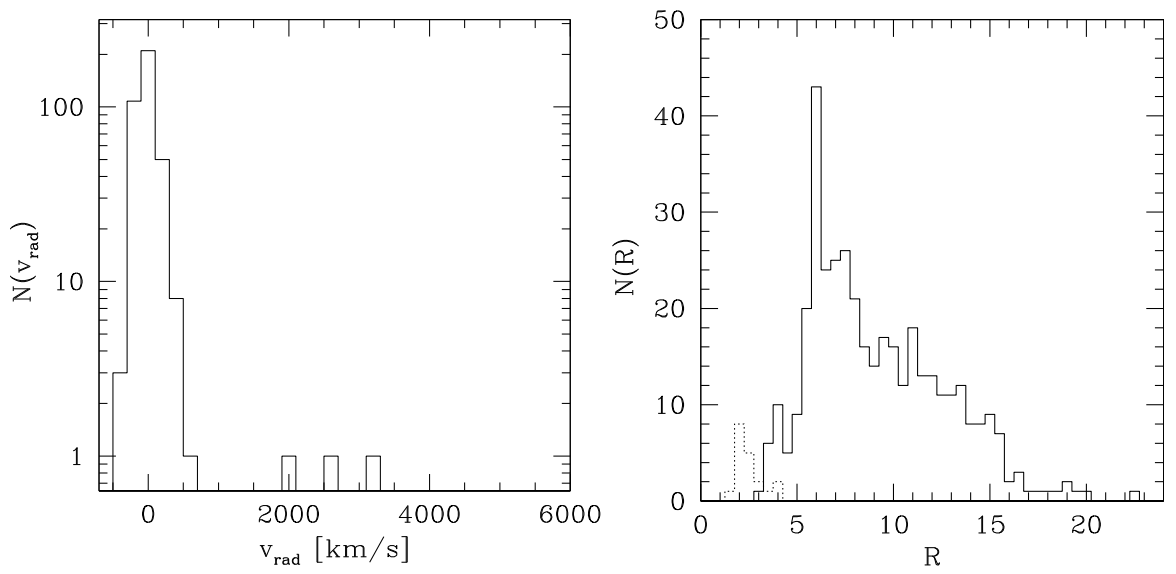

Fig. 6. Left panel: radial velocity histogram of the observed sources. The background regime $\left(>6000 \mathrm{~km} \mathrm{~s}^{-1}\right)$ is excluded. The three Centaurus cluster members are clearly distinguishable. Right panel: histogram of the confidence level $R$ achieved in the radial velocity measurement via cross-correlation with a template spectrum. The solid histogram indicates the sources with reliable measurement, the dotted histogram indicates sources without an acceptable cross-correlation result.

side, this remains within the $2 \sigma$ margin of uncertainty of the estimated number of UCDs. We note that the expected number of GCs drops below 0.5 only for $M_{V}<-12.8$ mag. From a purely statistical point of view, only the very brightest UCD luminosities $\left(M_{V} \simeq-13.5 \mathrm{mag}\right)$ are thus unaccounted for by a Gaussian GCLF.

With the database of Centaurus UCDs at hand, it is worthwhile to compare their spatial distribution to those in Fornax. In Fig. 7, we show the projected distance of these two UCD populations (with UCDs defined as compact stellar systems with $M_{V}<-11 \mathrm{mag}$ ) relative to the central galaxies of Centaurus and Fornax. The Fornax UCD database is the same as used in Mieske et al. (2008b). The distances are normalised to the $r_{500}$ radii of either cluster, for which Reiprich \& Boehringer (2002) measured $r_{500}=840 \mathrm{kpc}$ for Fornax, and $r_{500}=1.14 \mathrm{Mpc}$ for Centaurus. Within the radius of $120 \mathrm{kpc}$ surveyed for this publication, the Centaurus UCD population is slightly more clustered than the Fornax UCD population, at the $96.5 \%$ confidence level according to a KS-test. However, this may be at least partially because all Centaurus UCDs with $M_{V}>-12$ mag were discovered in our P76 survey, which had a more complete area coverage for $r<50 \mathrm{kpc}$ than for $r>50 \mathrm{kpc}$ (see Fig. 2). When restricted to $r<50 \mathrm{kpc}$ in Centaurus and a corresponding $r<35 \mathrm{kpc}$ in Fornax, the cumulative radial distribution of both samples is indistinguishable. When considering only UCDs with $M_{V}<-12 \mathrm{mag}$ - for which the spatial survey coverage is 


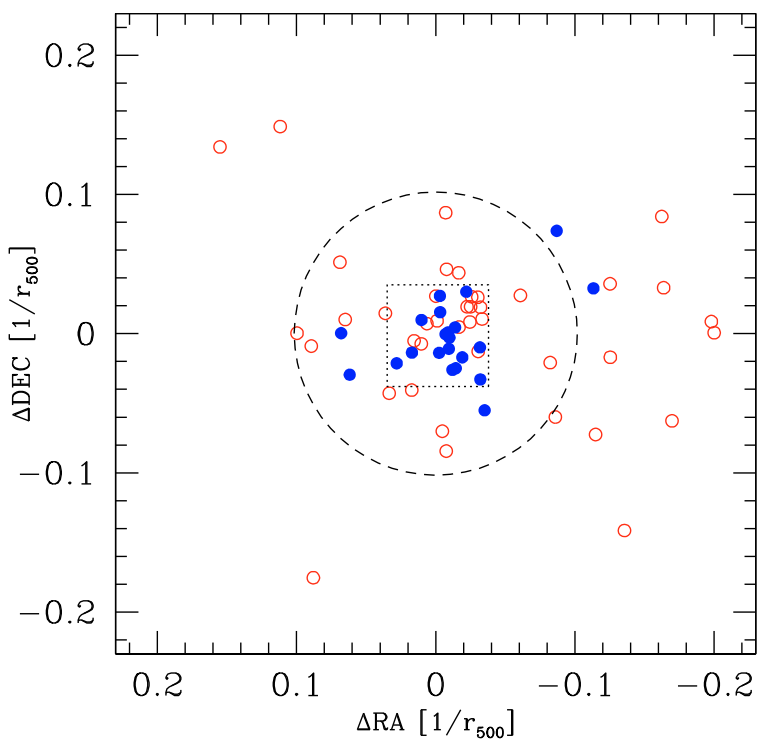

Fig. 7. Positions of confirmed UCDs in the Fornax (open red circles; Mieske et al. 2008b) and Centaurus clusters (filled blue circles; this paper and Mieske et al. 2007), relative to the central galaxies and normalised to the $r_{500}$ radius of the respective clusters. UCDs are selected as compact stellar systems with $M_{V}<-11 \mathrm{mag}$. The dashed circle indicates the coverage of the search for bright UCDs in Centaurus presented in this paper. The dotted square indicates the central quadrant of the P76 Centaurus survey (Mieske et al. 2007), within which the survey completeness was twice as high as outside (see also Fig. 3).

comparable between Fornax and Centaurus - we also find indistinguishable distributions according to a KS-test. We can therefore state that within $2 \sigma$, Fornax and Centaurus UCDs have the same radial distribution, when scaled to the respective $r_{500}$ radius of their host clusters.

There are a number of ongoing investigations of the UCD luminosity function towards very bright luminosities $\left(M_{V}<-12 \mathrm{mag}\right)$ in a range of environments, (e.g., the present work, Wehner \& Harris 2008; and Misgeld et al. 2008). It will be interesting to investigate how the luminosities and masses of the most massive UCDs correlate with the properties of their host environments (Hilker et al. 2009, in preparation), as previously studied in an analogous fashion for globular cluster systems
(Whitmore 2003; Larsen 2002) and systems of young massive clusters (e.g. Weidner et al. 2004).

\section{References}

Bertin, E., \& Arnouts, S. 1996, A\&AS, 117, 393

Chilingarian, I., \& Mamon, G. 2008, MNRAS, 385L, 83

Drinkwater, M. J., Jones, J. B., Gregg, M. D., \& Phillipps, S. 2000, PASA, 17, 227

Drinkwater, M. J., Gregg, M. D., Hilker, M., et al. 2003, Nature, 423, 519

Evstigneeva, E. A., Gregg, M. D., Drinkwater, M. J., \& Hilker, M. 2007, AJ, 133,1722

Fellhauer, M., \& Kroupa, P. 2002, MNRAS, 330, 642

Fellhauer, M., \& Kroupa, P. 2005, MNRAS, 359, 223

Firth, P., Drinkwater, M. J., Evstigneeva, E. A., et al. 2007, MNRAS, 382, 1342

Gebhardt, K., \& Kissler-Patig, M. 1999, AJ, 118, 1526

Haşegan, M., Jordán, A., Côté, P., et al. (VCS team) 2005, ApJ, 627, 203

Hilker, M., Infante, L., Vieira, G., Kissler-Patig, M., \& Richtler, T. 1999, A\&AS, 134,75

Hilker, M., Baumgardt, H., Infante, L., et al. 2007, A\&A, 463, 119

Jones, J. B., Drinkwater, M. J., Jurek, R., et al. 2006, AJ, 131, 312

Jordán, A., Blakeslee, J. P., Peng, E. W., et al. 2004, ApJS, 154, 509

Jordán, A., Côté, P., Blakeslee, J. P., et al. 2005, ApJ, 634, 1002

Jordán, A., McLaughlin, D. E., Côté, P., et al. 2006, ApJ, 651, L25

Jordán, A., McLaughlin, D. E., Côté, P., et al. 2007, ApJS, 171, 101

Kundu, A., \& Whitmore, B. 2001, AJ, 121, 2950

Larsen, S. S. 2002, AJ, 124, 1393

Larsen, S. S., Brodie, J. P., Huchra, J. P., Forbes, D. A., \& Grillmair, C. J. 2001, AJ, 121, 2974

Le Fevre, O., Saisse, M., Mancini, D., et al. 2003, SPIE, 4841, 1670

Mieske, S., Hilker, M., \& Infante, L. 2004, A\&A, 418, 445

Mieske, S., Hilker, M., \& Infante, L. 2005, A\&A, 438, 103

Mieske, S., Hilker, M., Jordán, A., Infante, L., \& Kissler-Patig, M. 2007, A\&A, 472, 111

Mieske, S., Hilker, M., Jordán, A., et al. 2008a, A\&A, 487, 921

Mieske, S., Hilker, M., Bomans, D. J., et al. 2008b, A\&A, 489, 1023

Misgeld, I., Mieske, S., \& Hilker, M. 2008, A\&A, 486, 697

Misgeld, I., Hilker, M., \& Mieske, S. 2009, A\&A, submitted

Phillipps, S., Drinkwater, M. J., Gregg, M. D., \& Jones, J. B. 2001, ApJ, 560, 201

Quintana, H., Ramirez, A., \& Way, M. J. 1996, AJ, 111, 603

Reiprich, T., \& Böhringer, H. 2002, ApJ, 567, 716

Rejkuba, M., Dubath, P., Minniti, D., \& Meylan, G. 2007, A\&A, 469, 147

Rhode, K. L., \& Zepf, S. E. 2001, AJ, 121, 210

Schlegel, D. J., Finkbeiner, D. P., \& Davis, M. 1998, ApJ, 500, 525

Thomas, P. A., Drinkwater, M. J., \& Evstigneeva, E. 2008, MNRAS, 389, 102

Tonry, J., \& Davis, M. 1979, AJ, 84, 1511

Wehner, E., \& Harris, W. 2008, ApJL, 668, 35

Weidner, C., Kroupa, P., \& Larsen, S. S. 2004, MNRAS, 350, 1503

Whitmore, B. 2003, in Extragalactic Globular Cluster Systems: Proceedings of the ESO Workshop Held in Garching, Germany, 27-30 August 2002, ESO Astrophys. Symp., ed. M. Kissler-Patig (Springer-Verlag), 336 